\title{
Primary Study on Mutation Effect in Taproots of Radish Cultivar "Huangzhou" by Ethylmethylsulfone (EMS)
}

\author{
Xiaomin $\mathrm{Yu}^{1}$, Changjun $\mathrm{Ge}^{2}$, Jianbo $\mathrm{Xu}^{3}$, Nana Zhang3, Shisheng $\mathrm{Li}^{3}$ \\ ${ }^{1}$ Journal of Huanggang Normal University, Huanggang, China \\ ${ }^{2}$ Huanggang Academy of Agricultural Science, Huanggang, China \\ ${ }^{3}$ Collaborative Innovation Center for the Characteristic Resources Exploitation of Dabie Mountains, \\ Key Laboratories of Economic Forest Germplasm Improvement and Comprehensive Resources \\ Utilization of Hubei Province, College of Life Science, Huanggang Normal University, Huanggang, China \\ Email: "21581872@qq.com
}

Received 15 February 2016; accepted 29 February 2016; published 4 March 2016

Copyright (C) 2016 by authors and OALib.

This work is licensed under the Creative Commons Attribution International License (CC BY). http://creativecommons.org/licenses/by/4.0/

(c) (i) Open Access

\section{Abstract}

Mutation breeding by Ethylmethylsulfone (EMS) has several advantages: high mutation ratio, many mutated phenotypes and low destruction which has been widely applied for crop breeding. We traced to uncover the mutagenic effect of EMS to Huangzhou radish by observation on germination ratio, root length and agronomic traits in field after soaking radish seeds by different concentration of EMS. In aspect of low germination ratio and short root length, the growth of radish is inhibited as the increasing concentration of EMS (from $0.2 \%$ to $2 \%$ ) which indicates the inhibited effect of EMS to "Huangzhou" radish. Furthermore, the suitable concentration of EMS for "Huangzhou" radish is $0.4 \%-0.8 \%$ according to the selection criteria of $50 \%$ lethal concentration. The field traits are recorded after the seed of "Huangzhou" treated by $0.5 \%$ EMS. Some agronomic traits on treated group were found including thickened taproots by compared with CK.

\section{Keywords}

Mutation, EMS, Radish, Thickened Taproots

Subject Areas: Agricultural Science

${ }^{*}$ Corresponding author.

How to cite this paper: Yu, X.M., Ge, C.J., Xu, J.B., Zhang, N.N. and Li, S.S. (2016) Primary Study on Mutation Effect in Taproots of Radish Cultivar "Huangzhou" by Ethylmethylsulfone (EMS). Open Access Library Journal, 3: e2420. 


\section{Introduction}

Radish (Raphanus Sativus L.) is a kind of cruciferous annual or biennial herb, which is a relative species of rape. As an important root vegetable crop in worldwide, radish has a very long history of planting. In the long-term evolution, cultivation and breeding process, radish's varieties are more abundant in the root shape and root color, leaf shape, leaf color and flavor, harvest and bolting stage, and other aspects of performance. With the expansion of the use of radish, it is becoming more and more important in people's daily life. It has gradually become the important object that many scholars carry out scientific research on radish [1]. At present, the research on development of radish is gradually enhanced, and the radish varieties are also growing. But it still cannot meet the huge demand for the economic and social needs nowadays. Conventional breeding methods are widely used in production, and the technology is easy to master, while the potential is hardly to be improved. As one of the modern biotechnology, mutation breeding is universally applied in animal and plant breeding [2]-[4]. It will be a great supplement to conventional breeding methods, and speeds up the process of crop breeding. In order to promote the radish breeding process, we carry out the chemical mutagen (Ethylmethylsulfone, EMS) studies on the mutagenic effect of taproots of radish. We aimed at providing an effective concentration of EMS for mutation on Huangzhou radish and obtaining a modified radish cultivar Huangzhou with new traits by EMS mutation. After soaping radish seeds by EMS, seed germination, root length of radish seedling, field characters are observed and analyzed. Finally, we found out the half lethal dose of EMS for radish cultivar Huangzhou and some new agronomic traits on Huangzhou radish, preliminary recognizing and understanding the mutagenic effects of EMS on radish taproots.

\section{Results}

\subsection{The Influence of EMS on the Germination Ratio of Huangzhou Radish}

According to the previous study in other crop treated by EMS, we selected 0, $0.2 \%, 0.4 \%, 0.6 \%, 0.8 \%, 1 \%, 2 \%$ EMS to soak Huangzhou radish seeds, then we measured the percentage of germination of Huangzhou seeds treated by different concentrations of EMS. Statistical results showed that the germination rate of Huangzhou radish seeds showed a gradual downward trend in the range of EMS 0.4\% - 2\% (Figure 1(a)), and the germination rate in the treatment of $2 \%$ EMS was 0.

\subsection{The Influence of EMS on the Root Growth of Huangzhou Radish}

After observation on the germination of Huangzhou seeds, we conducted a follow-up observation soon after statistics work about the root length of primary root which germinated within 7 days after seed soaking. The results showed that the growth of primary root was gradually inhibited with increase of concentration of EMS. The root length of primary root was significantly shorter when it was treated by $0.8 \%$ EMS (Figure 1(b)). The early seed germination and the primary root growth of radish seedlings were inhibited, and indicated that the treatment effect of EMS was persistent. Primary root length was significantly shortened and the length was less than 0.5 CM.
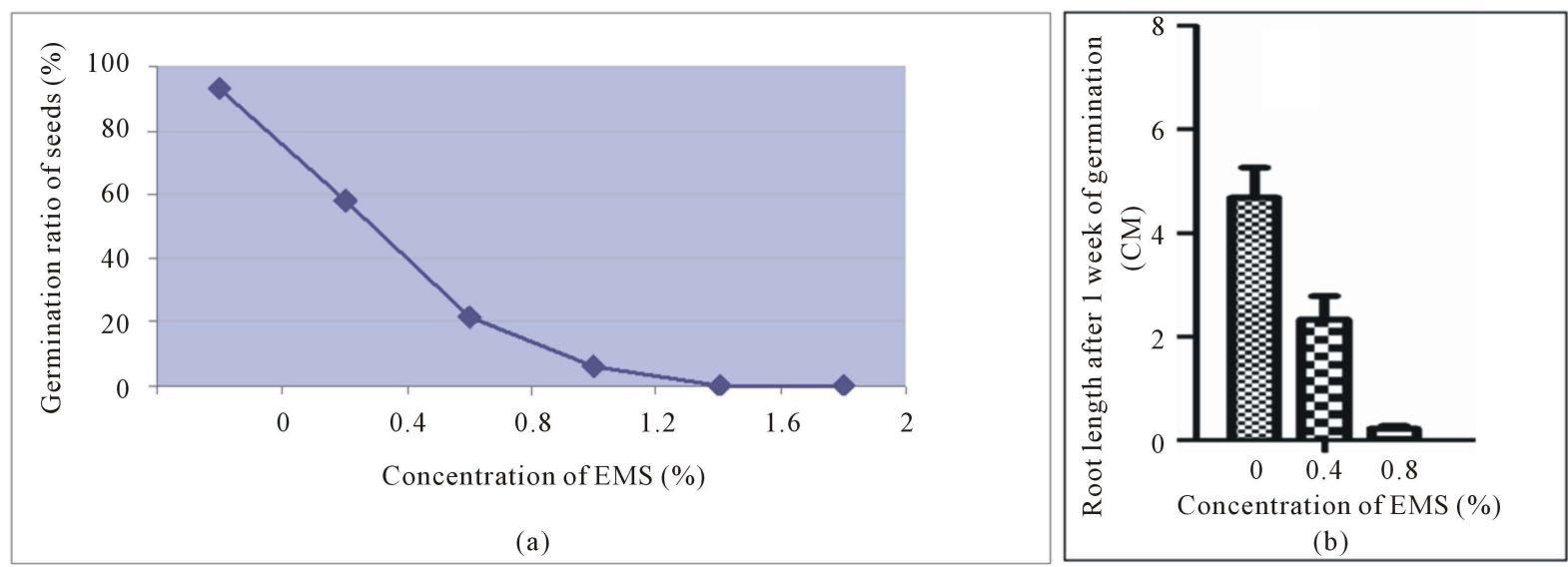

Figure 1. The effect of different concentrations of EMS on Huangzhou radish seed germination and root growth. 


\subsection{The Mutated Effect of EMS on Huangzhou in Abnormity}

According to seed germination rate and root length of seedlings, the half lethal dose of EMS was $0.4 \%-0.8 \%$ for Huangzhou radish. So we used the 0.5\% EMS mutation for a large number of Huangzhou radish seeds before sowing to field. Then the agronomic characters of Huangzhou radish treated by $0.5 \%$ EMS mutation were observed in continuation. We found numerous variations in a large number of mutants in Huangzhou radish. Taproots of Huangzhou were the focus of our study. The foundation of taproots of Huangzhou radish was originally flat at the bottom which showed an inverted triangle cone. After mutation, most taproots of Huangzhou were longer and thicker in contrast to normal Huangzhou (Figure 2). These variations were not the purpose of our original intention of mutation on Huangzhou radish. Via mutagenesis, we had planned to increase the proportion of the original characteristics of Huangzhou radish to obtain high purity of Huangzhou radish. The above test indicated that we obtained a considerable proportion of the Triangular shape and flat bottom of taproots in Huangzhou, while it still needed to be improved.

\section{Discussion}

Mutation breeding of ethyl methane sulfonate (EMS) had many advantages, such as high mutation frequency, high mutation character, little damage and it was widely used in a variety of crop breeding [5] [6]. The application of mutation breeding in radish had rarely been reported. In this study, the radish seeds were treated by different concentrations of EMS. EMS concentration (0.4\% - 0.6\%) was selected for the treatment of Huangzhou radish seeds, which laid a solid foundation for the establishment of the radish breeding. Han et al. (2007) reported the use of chemical reagent EMS on soybean mutation, and found that the mutagenic treatment concentration was $0.4 \%$. There was also a Chinese cabbage mutant library which was obtained by $0.4 \%$ EMS [4]. In wheat and rice mutant screening process, the suitable concentrations of EMS for wheat and rice were $1 \%$ and $1.5 \%$ respectively [7] [8]. It was not difficult to discover that there was difference in the sensitivity of EMS among different species. Combining with the results of above study, we also found that even in the same crop, different varieties had different sensitivity to EMS. Therefore, it was very important to choose the suitable EMS concentration to obtain the ideal mutagenic effect.

Using $0.5 \%$ EMS to deal with the radish cultivar Huangzhou, we found a series of abnormal traits in field. Han et al. (2007) found that there were obvious characters of the overlap of the cotyledon, three cotyledon and creeping growth in M2 generation of mutated soybean [9]. But in the M3 generation, the only creeping growth could be inherited which indicated that the mutagenic effect of this mutation could be inherited. The effect of genetic mutation was the ideal effect after EMS treatment. The mutagenic effects which couldn't be inherited by offspring only showed the certain drug effects of EMS on crops and they had no use for selection of mutants. Therefore, we would find a favorable and genetic mutation in the offspring of the treated radish. EMS could modify the sequence of DNA which was use to produce new phenotypes in crop [3] [5] [6]. The thicked taproot observed in Huangzhou radish population (Figure 2(b)) might due to lost function of some genes which were mutated by EMS, while the more details on the modified genes were absent in our study. The confirmation on function information of mutated genes in such Huangzhou population and the relationship between those mutated genes and phenotypes appeared in our radish population would be the next study object in our future work.

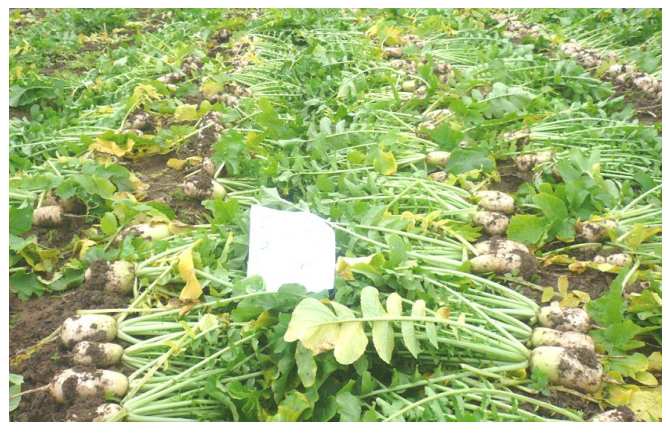

(a)

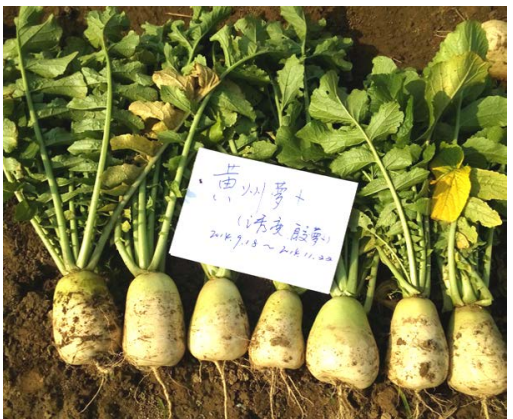

(b)

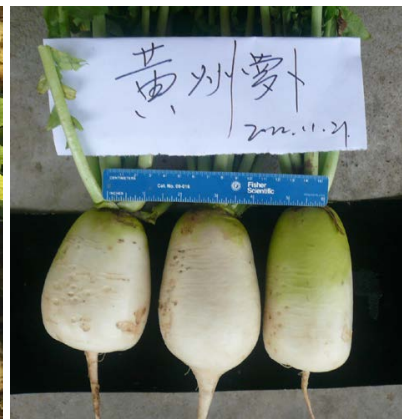

(c)

Figure 2. The thickened taproots in mutated Huangzhou M2 population with mutation frequency of 21.45\%. (a) Mutated Huangzhou radish in field; (b) Mutated taproots of Huangzhou radish; (c) Conventional Huangzhou radish. 


\section{Conclusion}

Mutated breeding is a conventional method as it can decrease the whole time on crop breeding. This was the first report on the application of EMS mutation in radish cultivar, Huangzhou. The suitable concentration on mutated breeding in radish by EMS was obtained and the mutated phenotypes of Huangzhou taproots were observed. 0.5\% EMS was selected for the treatment of Huangzhou radish seeds, which laid a solid foundation for the establishment of the radish breeding. Although the mutated taproots in Huangzhou were focused on the length of root which was not consistent with our study aim, the improvement on purity and quality of Huangzhou would be continued in the offspring of those mutated Huangzhou in our future work.

\section{Material and Methods}

\subsection{Material}

Radish varieties were purchased from Wuhan City Dongmen seed market.

\subsection{Observation on Growth of Seeds Treated by EMS}

One gram of radish seeds was taken and soaked using different concentrations of EMS (purchased from Sigma Company). The radish seeds were soaking by EMS before soaked 4 - 8 hours by water. Seeds were soaked by EMS under 120 rpm shaking and duration time for 4 hours. After being treated by EMS, soaked seeds were rinsed under water for 2 hours to remove residual EMS. Then treated seed by EMS and the control was placed in the 25 degree light incubator. After two days, the germination rate of the seeds treated by EMS was measured, and the root length of radish seed was measured after 7 days.

\subsection{Observation on Abnormity Trait}

Radish seeds were treated with EMS and cleaned before planting, abnormity traits were investigated from the true leaf stage to maturation.

\subsection{Data Analysis and Graphic Process}

The images were collected using image J (German) and data were analyzed by SPSS14.

\section{Acknowledgements}

This study was supported by Natural Science Fund of Hubei Province (2013CFC126), Innovation and entrepreneurship training program for college students (201510514027, SY201527) and Doctoral funding (2015002703) from Huanggang normal university

\section{Author Contributions}

Li shisheng designed the whole experiment, Li Shisheng, Yu Xiaomin, Ge Changjun, Xu Jianbo and Zhang Nana carried out the whole experiment, Yu Xiaomin and Li Shisheng analyzed the data, Li Shisheng wrote the paper.

\section{References}

[1] Kim, K.H., Moon, E., Kim, S.Y., Choi, S.U., Lee, J.H. and Lee, K.R. (2014) 4-Methylthio-butanyl Derivatives from the Seeds of Raphanus sativus and Their Biological Evaluation on Anti-Inflammatory and Antitumor Activities. Journal of Ethnopharmacology, 151, 503-508. http://dx.doi.org/10.1016/j.jep.2013.11.003

[2] Ahloowalia, B.S., Maluszynski, M. and Nichterlein, K. (2004) Global Impact of Mutation-Derived Varieties. Euphytica, 135, 187-204. http://dx.doi.org/10.1023/B:EUPH.0000014914.85465.4f

[3] Greene, E.A., Codomo, C.A., Taylor, N.E., Henikoff, J.G., Till, B.J., Reynolds, S.H., Enns, L.C., Burtner, C. and Johnson, J.E. (2003) Spectrum of Chemically Induced Mutations from a Large-Scale Reverse Genetic Screen in Arabidopsis. Genetics, 164, 731-740.

[4] Lu, Y., Liu, M.Y., Wang, S., Zhao, J.J., Wang, Y.H., Luo, S.X., Chen, X.P. and Shen, S.X. (2015) Effects of EMS Mutagenesis on Seed and Seedling Vigor and Phenotypic Variation of M2 Population in Chinese Cabbage. Journal of 
Plant Genetic Resource, 16, 349-358.

[5] An, X.L., Cai, Y.L., Wang, J.G., Wang, G.Q. and Sun, H.Y. (2003) Chemical Mutagen and Its Application on Plant Breeding. Acta Agriculture Nucleatae Sinica, 17, 239-242.

[6] Liang, R.F. and He, L.F. (2008) The Study Progress of Crop Breeding via EMS. Seed, 2, 47-49.

[7] Till, B.J., Cooper, J., Tai, T.H., Colowit, P., Greene, E.A., Henikoff, S. and Comai, L. (2007) Discovery of Chemically Induced Mutations in Rice by TILLING. BMC Plant Biology, 7, 19. http://dx.doi.org/10.1186/1471-2229-7-19

[8] Uauy, C., Paraiso, F., Colasuonno, P., Tran, R.K., Tsai, H., Berardi, S., Comai, L. and Dubcovsky, J. (2009) A Modified TILLING Approach to Detect Induced Mutations in Tetraploid and Hexaploid Wheat. BMC Plant Biology, 9, 115. http://dx.doi.org/10.1186/1471-2229-9-115

[9] Han, S.Y., Zhang, H.Y., Yang, M.L., Zhao, T.J., Gai, J.Y. and Yu, D.Y. (2007) Screening of Mutants and Construction of Mutant Population in Soybean “Nannong 86-4”. Acta Agronomica Sinica, 33, 2059-2062. 